\title{
The Relation Between Personality, Time Perspective and Positive Orientation in Chile, Hong Kong, and Poland
}

\author{
Aneta Przepiorka ${ }^{1}$ (D) Nicolson Yat-fan Siu ${ }^{2} \cdot$ Małgorzata Szcześniak $^{3}$. \\ Celina Timoszyk-Tomczak ${ }^{3}$ - Jacqueline Jiaying Le ${ }^{4} \cdot$ Mónica Pino Muñoz ${ }^{5}$
}

Published online: 25 April 2019

(C) The Author(s) 2019

\begin{abstract}
The objective of the present study was to examine the relationships between personality characteristics, time perspective (TP), and positive orientation (PO) across three countries representing different cultures: Chile, Hong Kong, and Poland. The participants were university students; they completed the Zimbardo time perspective inventory, the ten-item personality inventory, and the positive orientation scale. We investigated whether PO could be predicted by personality traits and TP. The study revealed that TP dimensions predicted PO even after controlling for personality traits. This effect was found in all three countries. Additionally, the study revealed the role of balanced time perspective as a moderator between personality traits and PO.
\end{abstract}

Keywords Time perspective $\cdot$ Balanaced time perspective $\cdot$ Positive orientation $\cdot$ Life satisfaction · Optimism · Self-esteem $\cdot$ Personality $\cdot$ Chile $\cdot$ Hong Kong $\cdot$ Poland

\section{Introduction}

Positive psychology puts strong emphasis on identifying the predispositions that contribute to happiness and enable people to function optimally in different life domains, such as work, school, or stress coping (e.g., Luthans et al. 2007). In the present study we extended this search by focusing more intensely on positive orientation (PO) and its relations with personality, time perspective (TP), and balanced time perspective (BTP).

PO is a construct related to self-esteem, life satisfaction, and dispositional optimism (Caprara et al. 2010). It refers to global positive evaluations of oneself, life, and the future.

Aneta Przepiorka

aneta.przepiorka@gmail.com; aneta.przepiorka@kul.pl

1 Institute of Psychology, The John Paul II Catholic University of Lublin, Al. Racławickie 14, 20-950 Lublin, Poland

2 Hong Kong Shue Yan University, North Point, Hong Kong

3 University of Szczecin, Szczecin, Poland

4 YMCA of Hong Kong Christian College, Tung Chung, Hong Kong

5 Universidad del Bío-Bío, Chillán, Chile 
These positive evaluations help individuals thrive and savor every moment of life despite encountering obstacles or experiencing difficult life conditions, despite the awareness of the unavoidable process of aging and inevitable death. Alessandri et al. (2012a) found that PO was related to intrapersonal and interpersonal areas of functioning and to psychological resilience. PO plays a crucial role in health promotion and has been found to be positively related, for instance, to smoking cessation (Grassi et al. 2014). PO has proved to be stable across cultures and unidimensional (Alessandri et al. 2012b; Caprara et al. 2010; Heikamp et al. 2014).

In our study we used data from Chile, Hong Kong, and Poland-countries with different cultural backgrounds, representing three different continents (Latin America, East Asia, and Central Europe) - in order to investigate the relationships of PO with TP and personality. The rationale for the choice of these three countries for comparison is presented further, in the Present Study section. In our study, we wanted, firstly, to verify whether PO could be predicted from personality traits and TP. Secondly, we wanted to examine the moderating role of BTP between personality traits and PO. Why can the relationships between personality, TP, and PO be relevant to examine? To answer this question, let us look more closely at each of these constructs and at the interrelations among them.

\subsection{Time Perspective and Balanced Time Perspective}

The way people perceive time may be important for their optimal functioning. In the present study, we explore PO drawing on Zimbardo's TP theory as one of the best-established theories addressing TP issues across different cultures (Sircova et al. 2014). According to Zimbardo and Boyd, TP is "the often non-conscious process whereby the continual flows of personal and social experiences are assigned to temporal categories, or time frames, that help to give order, coherence, and meaning to those events" (1999, p. 1271). It exerts a strong impact on human activity, motivation, and planning (Boniwell and Zimbardo 2004).

Zimbardo and Boyd (1999) distinguished five TPs: Past Positive, Past Negative, Present Fatalistic, Present Hedonistic, and Future. Each TP has its own associated benefits and costs. People with a dominant Past Positive TP have a tendency to recollect pleasant events from the past and to positively evaluate what happened. Memories give people a sense of continuity, and the related emotions allow them to reinterpret experiences in a more positive way (Shmotkin and Shrira 2012). In a study by Garcia (2014), the subjects who remembered more positive life events than negative ones were happier. What is more, Past Positive temporal frame had beneficial effects on well-being (Bhullar et al. 2015). People with a Past Positive TP focus on the good days, nostalgia, gratitude, and past successes. There is no doubt that they will exhibit a more sentimental and positive evaluation of their past, which is related to the core construct of PO. Previous research has also reported that Past Positive TP is related to high self-esteem and happiness (Zimbardo and Boyd 1999). Individuals with a dominant Past Negative TP often think back to the negatively evaluated past and have a tendency to recollect disagreeable situations. Past Negative TP was particularly indicative of psychiatric problems (van Beek et al. 2011). Participants with Past Negative orientation would generally hold a negative and aversive view of the past, in which they may have experienced unpleasant or traumatic events (Zimbardo and Boyd 1999; Sword et al. 2014). Research has also found that Past Negative orientation is related to low self-esteem and low emotional stability (Stolarski et al. 2015). Similarly, other studies (Sailer et al. 2014) revealed negative correlations of happiness with Past Negative TP. In the case of Present Hedonistic TP, the 
dominant tendency is to focus on pleasure "here and now," without considering the consequences or past experiences into account. In the study by Sailer et al. (2014), Present Hedonistic TP was related to happy feelings and high boredom avoidance. In a different study, by Desmyter and De Raedt (2012), Present Hedonistic TP was related to high positive affect. Present Fatalistic TP consists in passive existence in the present, stemming from the belief that one has no influence on what will happen in the future. Higher hopelessness and higher pessimistic view about the present were associated with lower levels of psychological well-being and positive affect (Sailer et al. 2014). Future TP is connected with a tendency to make plans, formulate goals, and consider the future consequences of present behaviors (Zimbardo and Boyd 1999, 2008). Future TP has been found to be a positive predictor of health behaviors (Mahon et al. 1997) and entrepreneurial initiative (Przepiorka 2016), as well as a negative predictor of behaviors such as smoking (Jones et al. 2009), eating disorders or drinking problems (Laghi et al. 2012), and risky driving (Zimbardo et al. 1997).

Recently, a construct called balanced TP (BTP has been introduced, defined as a the most adaptive TP profile (Stolarski et al. 2011a, b; Zimbardo and Boyd 1999). It is operationalized as a combination of optimal scores on different TPs. Having a BTP helps an individual to switch flexibly between past, present, and future in response to situations and to select adaptive TPs (Past Positive, Present Hedonistic, and Future) occasionally supplemented by maladaptive time horizons (Past Negative and Present Fatalistic) (Zimbardo and Boyd 2008). BTP has been found to be a positive predictor of well-being (e.g., Osin et al. 2009; Stolarski 2016; Zhang et al. 2013). BTP individuals are more satisfied and experience more positive affect in life (i.e., Ivanchenko et al. 2009). In previous studies, BTP functioned as a moderator as well as a mediator between different measures of emotional well-being and personality traits.

For instance, it has been found that BTP moderates the relationship between wellbeing and two personality traits: extraversion and neuroticism (Stolarski 2016). In that study, the author used a construct called Deviation from BTP (DBTP). The author found that DBTP predicted well-being when personality traits were controlled for. In that model, extraversion and neuroticism were significant predictors of well-being. It should also be noted that there was a positive association between DBTP and neuroticism and a negative associations with extraversion. At a low level of DBTP, the level of happiness was above average, minimizing the effects of the personality traits. In other study, the mediating role of unbalanced TP (DBTP-M; here the index was extended by Carpe Diem Scale) was significant for neuroticism and all measures of well-being (SobolKwapinska 2016). The mediating effect of DBTP-M was the highest for the relationship between neuroticism and life engagement. According to the data presented by Stolarski et al. (2016), BTP was a mediator between mindfulness and life satisfaction. Other previous research has also examined the interesting relationship between PO and BTP (Sobol-Kwapinska and Jankowski 2016). The authors used canonical correlations to verify whether two groups of variables forming PO and TP correlated with each other. They prepared two sets of variables: one consisted of self-esteem, satisfaction with life, and optimism, and the second comprised the specific ZTPI scales. They found that the combination of high Past Positive, moderate Future, low Present Fatalistic, and Negative Past orientations was strongly and positively correlated with PO. The structure of factor loadings was similar to the structure of BTP (Zimbardo and Boyd 2008). As PO was a predictor of a set of temporal variables dominated mostly by low Past Negative and Present Fatalistic perspectives, the authors pointed out that PO was a general positive attitude towards oneself, while BTP was a general positive attitude towards time 
with an emphasis on the role of the present (Sobol-Kwapinska and Jankowski 2016). Only Present Hedonistic perspective was significant in neither of the sets of variables: neither in the temporal ones related to PO, nor in the one related to BTP.

\subsection{Positive Orientation, Personality, and Time Perspective}

Personality is generally referred to as an individual's relatively stable traits and unique characteristics that contribute to consistent patterns of thoughts, emotions, and behavior (Funder Funder 1997; Michel et al. 2004). Personality is one of the most important predictors of life satisfaction (e.g., Boyce et al. 2013; Joshanloo and Afshari 2011). A meta-analysis of the relationships between the Big Five personality dimensions and subjective well-being, reporting that conscientiousness is positively associated with life satisfaction and positive affect and inversely related to negative affect (Steel et al. 2008). Similarly, conscientiousness has been found to be positively associated with self-esteem (Robins et al. 2001) and optimism (Sharpe et al. 2011).

In the literature there are some indications of links between personality and PO. A positive association was found between PO and extraversion, emotional stability, agreeableness, conscientiousness, and openness to experience (Caprara et al. 2012). PO correlated positively with energy, agreeableness, emotional stability, conscientiousness, and openness to experience measured with the Big Five Questionnaire (Caprara et al. 2012). In a Polish study, PO showed an associations with the Five-Factor Model (Miciuk et al. 2016). The authors also found a positive relationship of PO with extraversion and conscientiousness, and a negative relationship between PO and neuroticism; correlations of PO with openness and agreeableness were not found.

While testing the link between TP and personality, Kairys and Liniauskaite (2015) found correlations between TPs and the Big Five dimensions; more specifically, they found relationships between Future TP and Conscientiousness (.60), between Past Negative TP and Neuroticism (.48), and between Present Hedonistic TP and Extraversion (.30). Personality problems are usually correlated with Past Positive, Past Negative, and Present Fatalistic TPs.

Other study by Zhang and Howell (2011) revealed that neuroticism as a tendency to experience negative affects correlated significantly and strongly with Past Negative TP, and less strongly with Present Fatalistic and low Present Hedonistic TPs, whereas extraversion regarded as a positive emotionality correlated significantly with high Past Positive and Present Hedonistic TPs and with low Past Negative TP. Conscientiousness correlated with high Future TP and, to a smaller degree, with low Past Negative and Present Fatalistic TPs.

Previous findings showed that TP predicted life satisfaction over and above the Big Five personality traits (Stolarski and Matthews 2016; Zhang and Howell 2011). Stolarski and Matthews (2016) found that TP predicted mood states. In their study and in the one by Zhang and Howell (2011), TP added $11 \%$ and 14\%, respectively, to the explained variance in life satisfaction beyond the Big Five traits, whereas personality traits explained an additional $4 \%$ of variance in life satisfaction beyond TPs in both studies. Zhang and Howell (2011) underlined the role of Past Negative and Past Positive TPs in explaining the relationships between neuroticism and life satisfaction (a combination that explained $50 \%$ of the link). All these findings argue that TP as a construct cannot be easily reduced to personality and stands over and above the Big Five (Matthews and Stolarski 2015). 


\section{The Present Study}

Based on the findings presented, we assumed that TP dimensions would predict PO (H1) even after controlling for personality traits. Additionally, we set out to investigate the role of DBTP in the relationship between personality traits and PO. We wanted to examine the role of DBTP and to test whether acts as a moderator of the relationship between personality traits and PO $(\mathrm{H} 2)$. In the role of moderator, DBTP determines the conditions when personality traits influence PO (cf. Baron and Kenny 1986).

Why can BTP play a significant role in the relationship between personality traits and PO? Previous studies show that BTP performs also an important function in regulating emotions (for a review, see Matthews and Stolarski 2015). BTP correlated at .31 with overall emotional intelligence score (Stolarski et al. 2011a, b), which may suggest a similarity between BTP and EI as competency in monitoring emotional state. Given the link between personality traits and emotions (Oatley et al. 1996), the way we cope with our emotionality is related to our functioning in various domains (Gross 2008) and, consequently, with our evaluation of our life or ourselves. What specific forms of emotion regulation we use does not depend exclusively on personality traits but also on other factors, including BTP. BTP is associated with the choice of the type of memories, with planning, and with the anticipation of events; what is more, it modulates - to various degrees - the strategies of coping with stress (Matthews and Stolarski 2015), which may moderate the influence of personality on the way we evaluate our life and experience and on the way we perceive the future.

The choice of countries to be included in this study was determined by differences on personality dimensions (e.g., McCrae and Terracciano 2005) and by differences in the hierarchy of values (Schwartz 2004). The indices of quality of life, economic growth, globalization, and Gross Domestic Product per capita in Chile, Hong Kong, and Poland show that these countries are in the phase of development (CIA, 2015). According to the World Bank (2017), they differed in terms of GDP per capita (current US\$) as follows (for 2010-2014): Chile-15,732.3; Hong Kong-38,123.5; Poland-13,648.0. In the World Database of Happiness, the average happiness score (on a scale from 0 to 10) was 6.7 for Chile, 6.6 for Hong Kong, and 6.4 for Poland (Veenhoven 2015). In July 2014, Chile had 17,363,894 inhabitants, Hong Kong was inhabited by 7,112,688 people, and Poland had a population of $38,346,279$. Each of the three countries had a difficult history behind it, with communism, totalitarianism, or dictatorial governments. Due to some historical perturbations and the types of political system they have experienced, all these countries have retained some aspects of collectivistic culture, which involves a tendency for people to perceive themselves from a sociocentric perspective-as having group-related rather than personal goals and as being interdependent (Markus and Kitayama 2003; Oyserman et al. 2002). On the other hand, individualistic culture also played a role in each of these three countries. Although Chile was classified as predominantly collectivistic in the past, recent research has indicated a different self-construal in Chilean people, who scored higher on independence than on interdependence (e.g., Kolstad and Horpestad 2009). Hong Kong was one of Britain's colonies for a century; individualism was promoted and spread there (e.g., Hwang et al. 2003). Finally, in Poland-which is one of the European countries-the rights and privileges of individuals have been emphasized (e.g., Forbes et al. 2009). Each of these three countries represents a different composite mix of collectivism and individualism. They also have different dominant religions (an eclectic mixture of local religions in Hong Kong and Roman Catholicism in Poland and Chile). In a previous study presenting the worldwide distribution of personality traits (Schmitt et al. 2007), these three countries were 
Table 1 Demographic information

\begin{tabular}{llll}
\hline & $\begin{array}{l}\text { Chile } \\
N=301\end{array}$ & $\begin{array}{l}\text { Hong Kong } \\
N=203\end{array}$ & $\begin{array}{l}\text { Poland } \\
N=306\end{array}$ \\
\hline $\begin{array}{llll}\text { Gender } \\
\text { Female }\end{array}$ & $171(57 \%)$ & $143(70 \%)$ & $209(68 \%)$ \\
Male & $128(43 \%)$ & $60(30 \%)$ & $97(32 \%)$ \\
Age & & & \\
Mean $(S D)$ & $19.7(2.17)$ & $20.5(2.08)$ & $21.56(2.05)$ \\
Range & $17-37$ & $17-31$ & $19-30$ \\
Education & & & \\
1st year & $50.3 \%$ & $32.5 \%$ & $46.4 \%$ \\
2nd year & $13.3 \%$ & $36.0 \%$ & $27.5 \%$ \\
3rd year & $22.0 \%$ & $18.2 \%$ & $20.1 \%$ \\
4th year and above & $14.0 \%$ & $7.9 \%$ & $6.0 \%$ \\
\hline
\end{tabular}

classified as manifesting some cross-cultural differences. Based on the study by Hofstede (2001), the individualism-collectivism estimate is 23 for Chile, 25 for Hong Kong, and 60 for Poland. The higher the score, the higher the level of individualism. Chile is a relatively collectivistic country in the West, while Hong Kong is a relatively collectivistic one in the East. Poland is a relatively individualistic country.

\section{Method}

\subsection{Participants}

In each country (Chile, Hong Kong, Poland), undergraduate university students were invited to participate in the study. A total of 810 participants were recruited: 301 from Chile, 203 from Hong Kong, and 306 from Poland (see Table 1 for details).

\subsection{Procedure}

We collected data in Chile, Poland, and Hong Kong concurrently. The recruitment procedure was similar in the three groups. Informed consents were first obtained from the participants, who they were then instructed to complete the questionnaire. The participants were approached in a big lecture hall at their universities and received no remuneration for their participation. They were informed about the general aim of the study and assured of its anonymity. Twenty-one participants from all groups did not return the questionnaires and were regarded as withdrawals. The research project received approval from the institutional review board of the John Paul II Catholic University of Lublin.

\subsection{Measures}

The questionnaire administered in the study comprised three measures: the Zimbardo time perspective inventory, the ten-item personality inventory, and the positive orientation scale. The measures that were not available in the native languages of Chile (Spanish), Poland 
Table 2 Tucker's Phi coefficients comparing the factor structure in each country to the pooled solution

\begin{tabular}{llll}
\hline & \multicolumn{2}{l}{ Tucker's phi } \\
\cline { 2 - 4 } & Hong Kong & Chile & Poland \\
\hline Positive orientation & .78 & .83 & .96 \\
Past Negative & .95 & .92 & .97 \\
Present Hedonistic & .97 & .99 & .98 \\
Future & .97 & .99 & .99 \\
Past Positive & .96 & .95 & .94 \\
Present Fatalistic & .95 & .83 & .93 \\
\hline
\end{tabular}

(Polish), and Hong Kong (Traditional Chinese) were first translated from the English version. Then the scales were back-translated into English by another research assistant. The discrepancies identified between the original version and the translated version were resolved, and the problematic items underwent the process of translation and back-translation again until there were no more discrepancies, which was meant to ensure the validity and reliability of the translation of the original measure.

To assess the factor structure equivalence of the instruments used, we computed Tucker's phi coefficients using an Excel spreadsheet containing the appropriate formula. By all conventions, Tucker's phi (see Table 2) indicated a good cross-cultural equivalence of the PO and the ZTPI (Lorenzo-Seva and Berge 2006). ${ }^{1}$

\subsubsection{The Zimbardo Time Perspective Inventory (ZTPI)}

To measure TP, we administered the 56-item ZTPI (e.g., Zimbardo and Boyd 1999). The instrument includes five scales, corresponding to five TPs: Past Negative (Cronbach's $\alpha=.83$ in Poland, .79 in Chile, and .77 in Hong Kong), Past Positive (Cronbach's $\alpha=.76$ in Poland, .87 in Chile, and .72 in Hong Kong), Present Hedonistic (Cronbach's $\alpha=.81$ in Poland, .80 in Chile, and .73 in Hong Kong), Present Fatalistic (Cronbach's $\alpha=.84$ in Poland, .87 in Chile, and .71 in Hong Kong), and Future (Cronbach's $\alpha=.78$ in Poland, .73 in Chile, and .70 in Hong Kong). Responses were given on a 5-point Likert scale, from 1 (very untrue) to 5 (very true). In the present study we used the adapted versions of this measure: Spanish (Diaz-Morales 2006), Chinese (Wang et al. 2015), and Polish (SobolKwapinska et al. 2016).

In order to investigate the role of optimal TP, we assessed the level of BTP using the DBTP index proposed by Stolarski et al. $(2011 \mathrm{a}, \mathrm{b})$. We calculated the root of the squared deviations of individuals' scores from the optimal score on each ZTPI dimension. DBTP values close to zero indicate almost perfect BTP. Positive values indicate the extent to which a given individual's TP is far from optimal. The higher the DBTP index, the less balanced the TP. We followed the formula presented by Zhang et al. (2013).

\footnotetext{
1 The Ten-Item Personality Inventory was not subjected to this analysis because it contains only two items in each subscale and therefore factor analyses could not be applied.
} 


\subsubsection{Ten-Item Personality Inventory (TIPI)}

For personality measurement, we used the ten-item personality inventory (TIPI). TIPI is a ten-item measure (e.g., Gosling et al. 2003). It measures the following personality traits: extraversion, agreeableness, conscientiousness, emotional stability, and openness to experience. The values of Cronbach's $\alpha$ ranged from .46 to .84 in the three countries. They were the lowest for the Openness to Experience scale and the highest for the Agreeableness scale. The items are rated on a 7-point Likert scale from 1 (disagree strongly) to 7 (agree strongly). We administered the adapted versions of this measure: Spanish (Renau et al. 2013), Chinese (Huang et al. 2014), and Polish (Laguna et al. 2014).

\subsubsection{Positive Orientation Scale (POS)}

To measure positive orientation, we used the positive orientation scale (POS; Caprara et al. 2012). This scale is an 8-item instrument. Items are rated on a 5-point Likert scale ranging from 1 (strongly disagree) to 5 (strongly agree). As regards the reliability of this scale in our study, Cronbach's $\alpha$ was .73 in Poland, .85 in Chile, and .84 in Hong Kong. A Polish adaptation of this measure had already been published by Laguna et al. (2011), and the authors of the present study translated the scale into the other two languages (Spanish and Chinese).

\section{Results}

To assess the relationship of PO with TP and personality, we computed Pearson's $r$ correlations. The descriptive statistics and intercorrelations among the variables for the samples representing the three countries are presented in Tables 3 and 4.

In Chile (Table 3, below diagonal), those who scored high on PO were more Future-oriented, viewed their past more positively, and were more extraverted, more agreeable, more conscientious, more emotionally stable, as well as more open to new experience. Additionally, the individuals who scored lower on PO had more Past Negative experiences and a less balanced TP.

In Hong Kong (Table 3, above diagonal) those who scored high on PO had higher Present Hedonistic TP, were more Future-oriented, viewed their past in a more positive light, and focused less fatalistically on the present. They scored higher on BTP. They were also more extraverted, conscientious, emotionally stable, and open to new experience.

In Poland (Table 4), those with higher PO focused more hedonistically on the present and viewed their past more positively. They scored lower on Past Negative and Present Fatalistic TPs. They were more extraverted, agreeable, conscientious, emotionally stable, and open to new experience. There scored higher on BTP.

There were some gender differences across the three countries. We compared mean differences using independent samples $t$-tests. The magnitude of differences was measured using Cohen's $d$ effect size for unequal sample sizes and interpreted using Cohen's (1988) guidelines: small $d=0.2-0.5$, moderate $d=0.5-0.8$, and large $d>0.8$. In Chile there was a difference in Present Hedonistic TP, $t(297)=-3.572, p=.000, d=0.42$, with men scoring higher $(M=3.42, S D=0.52)$ than women $(M=3.20, S D=0.54)$, and in Past Positive TP, $t(297)=-2.087, p=.038, d=0.24$, with men scoring higher 


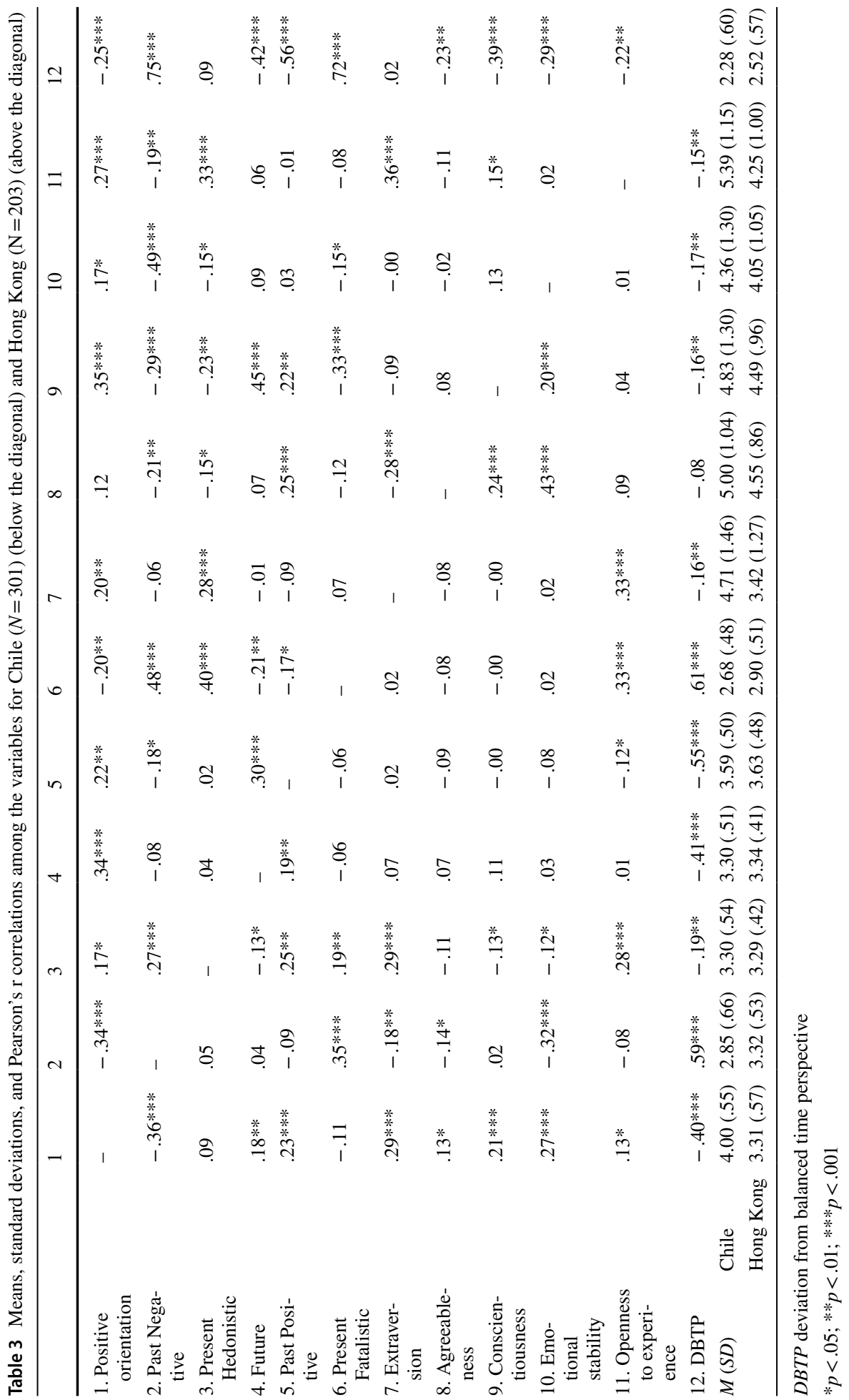




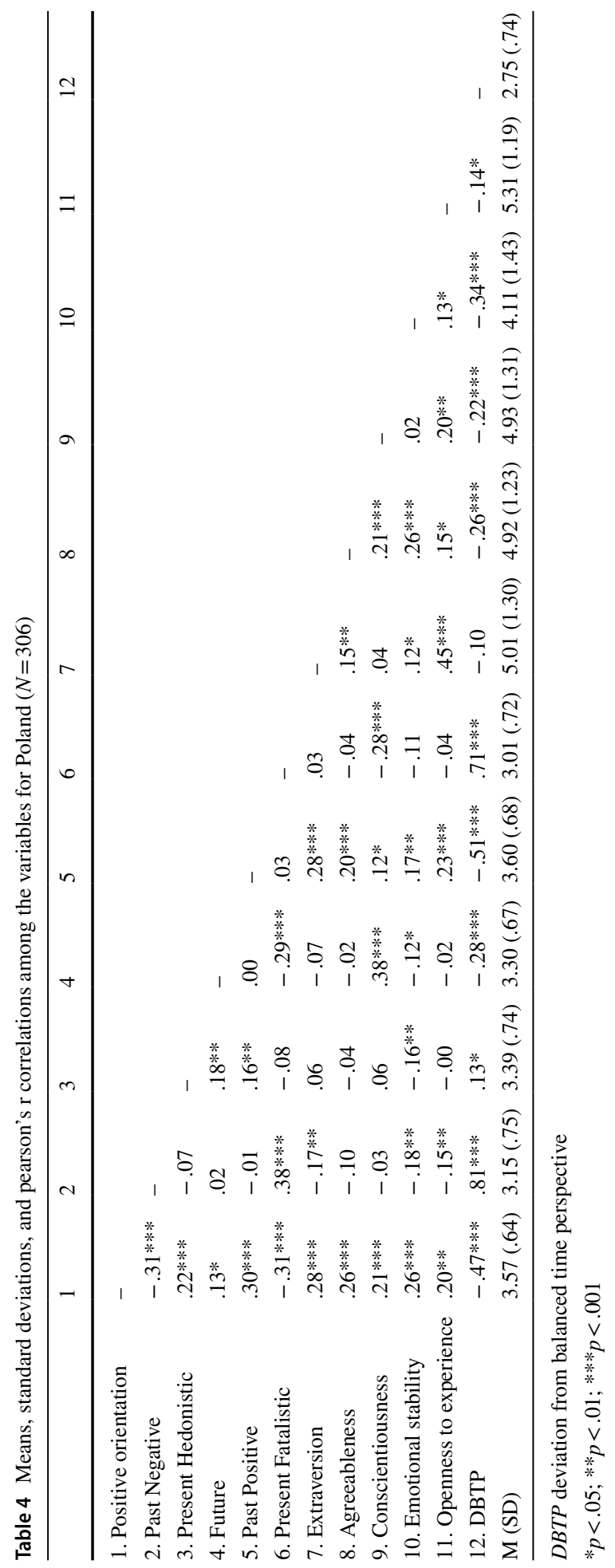


$(M=3.66, S D=0.47)$ than women $(M=3.54, S D=0.51)$. In Hong Kong there were differences in Past Negative TP, $t(201)=-3.543, p=.000, d=0.57$, with men $(M=3.51$, $S D=0.44)$ scoring higher than women $(M=3.23, S D=0.54)$; in Present Fatalistic TP, $t(201)=-2.355, p=.019, d=0.35$, with men $(M=3.03, S D=0.53)$ scoring higher than women $(M=2.85, S D=0.50)$; in DBTP $t(201)=-2.616, p=.010, d=0.41$, with men $(M=2.68, S D=0.54)$ scoring higher than women $(M=2.45, S D=0.57)$; and in agreeableness, $t(201)=4.319, p=.000, d=0.68$, with women $(M=4.71, S D=0.86)$ scoring higher than men $(M=4.17, S D=0.71)$. In Poland there was a difference in emotional stability, $t(304)=-2.023, d=0.23$, with men $(M=4.11, S D=1.14)$ scoring higher than women $(M=3.82, S D=1.34)$.

\subsection{Regression Analyses}

We ran several hierarchical regression analyses in order to determine the predictive power of TPs and the Big Five traits on PO according to the procedure applied by Stolarski and Matthews (2016) to test Hypotheses 1. Tables 5 and 6 present the results of regression analyses for the three countries (Chile, Hong Kong, and Poland) separately. In the first step of the analyses, we entered TPs or personality traits; in the second step, we entered the remaining set of variables: TPs if personality traits had been entered in the first step, and personality traits if TPs had been entered first.

The results show that the regression model was significant when TPs were entered in Step 1 and personality traits were added in Step 2 (Table 5). In Step 1, the scores on TP scales explained 19\%, 11\%, and $48 \%$ of variance in PO in Chile, Hong Kong, and Poland, respectively. The inclusion of the Big Five in Step 2 added an extra 8\%, 6\%, and $5 \%$ to these values.

In the case of Chile, Past Positive, Past Negative, and Future TPs turned out to be significant predictors of PO in Step 2. As regards personality traits, extraversion, conscientiousness, and emotional stability were positive predictors of PO. In the case of Hong Kong, Present Hedonistic TP was a significant predictor of PO in Step 2. In the case of Poland, in Step 2, Past Positive, Past Negative, and Present Hedonistic TPs were significant predictors of PO. Of the personality traits, only extraversion and emotional stability significantly contributed to PO.

Next, we reversed the sequence of the variables (Table 6) by entering the Big Five traits in the first step and TPs in the second step. The Big Five explained 17\%, 12\%, and $27 \%$ of the variance in PO in Chile, Hong Kong, and Poland, respectively, and TPs explained additional $10 \%, 4 \%$, and $24 \%$, which suggests that TP has incremental validity in predicting PO over the Big Five in the case of Chile and Poland.

In Chile, Past Negative, Past Positive, and Future TPs were significant negative predictors of PO in Step 2. Extraversion, conscientiousness, and emotional stability had significant $\beta$ value of PO. The strongest predictor of PO was Past Negative TP. In Hong Kong, Present Hedonistic TP and conscientiousness significantly predicted PO in Step 2. The strongest predictor of PO was conscientiousness. In Poland, Present Hedonistic TP, Past Positive TP, extraversion, emotional stability, and agreeableness were significant positive predictors of PO, whereas Past Negative TP was a significant negative predictor of PO in Step 2. The strongest predictor of PO was Past Positive TP. 


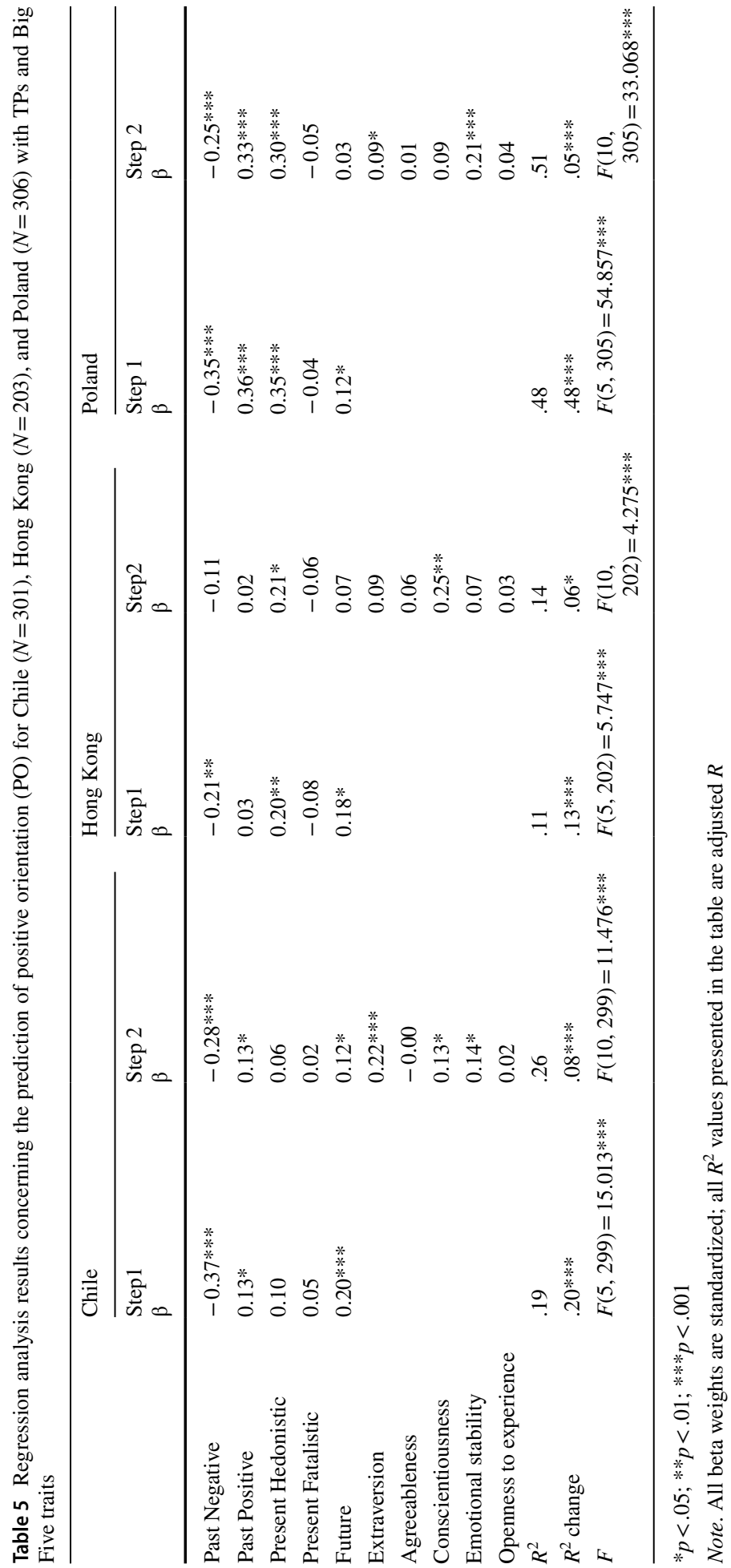




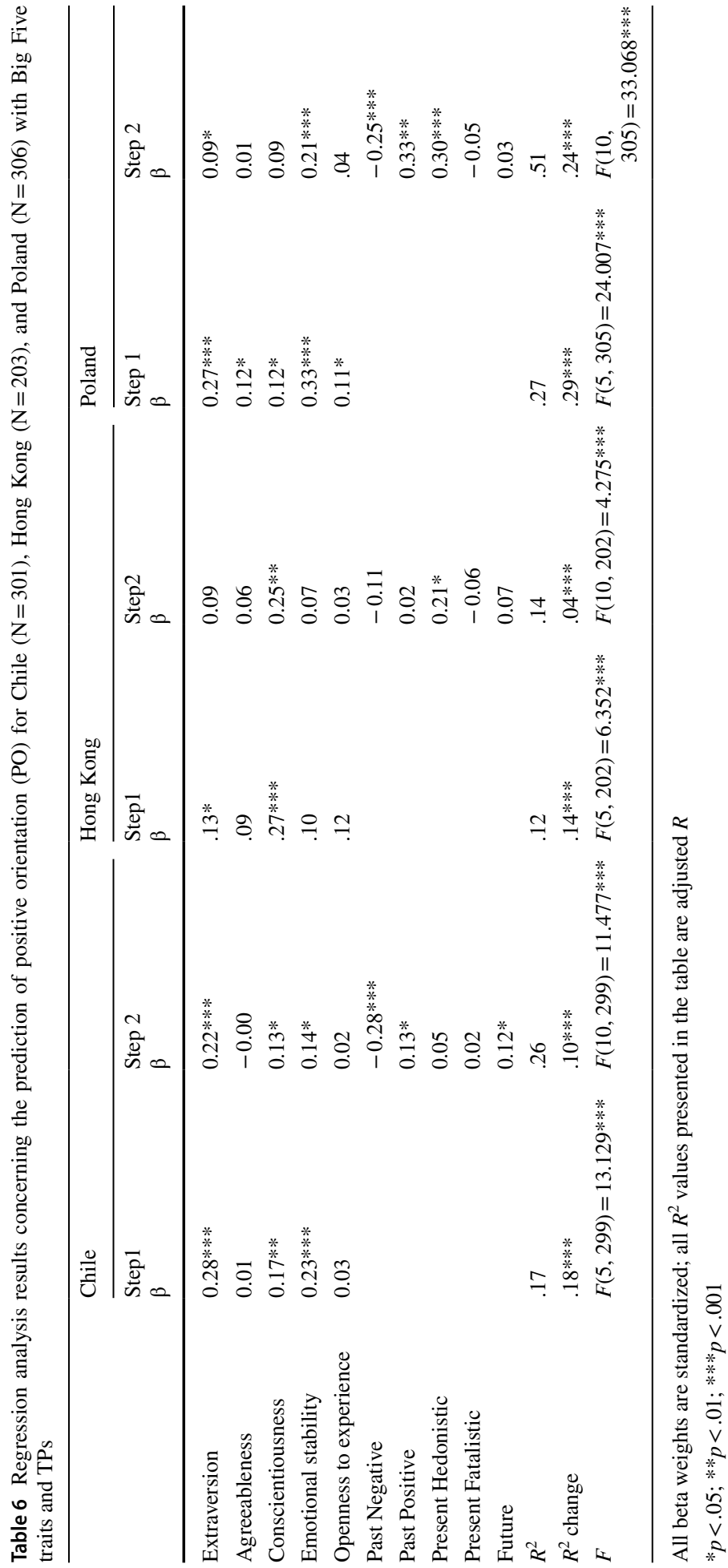


Table 7 The results of moderation analysis for the positive orientation dependent variable; moderatorunbalanced TP (DBTP)

\begin{tabular}{|c|c|c|c|c|c|c|c|}
\hline Country & Predictor & $R_{\mathrm{ch}}^{2}$ & $B$ & $t$ & $p$ & $95 \% \mathrm{CI}$ & $\begin{array}{l}\text { JN-John- } \\
\text { son-Neyman } \\
\text { cutpoint }\end{array}$ \\
\hline \multirow[t]{5}{*}{ Hong-Kong $n=203$} & Extraversion & .01 & -.03 & 1.08 & .282 & -.03 to .10 & \\
\hline & Agreeableness & .01 & -.10 & -1.71 & .089 & -.22 to .02 & \\
\hline & Conscientiousness & $<.01$ & -.00 & -0.04 & .968 & -.09 to .09 & \\
\hline & Emotional stability & .01 & -.05 & -1.13 & .262 & -.13 to .04 & \\
\hline & Openness to experience & .02 & .08 & 1.93 & .055 & .00 to .17 & \\
\hline \multirow[t]{5}{*}{ Chile $n=300$} & Extraversion & .03 & .10 & 3.40 & .001 & .04 to .16 & -.38 \\
\hline & Agreeableness & $<.01$ & .02 & 0.37 & .714 & -.07 to .10 & \\
\hline & Conscientiousness & $<.01$ & .07 & 0.22 & .828 & -.07 to .08 & \\
\hline & Emotional stability & .01 & .04 & 1.33 & .185 & -.02 to .11 & \\
\hline & Openness to experience & .01 & .08 & 2.16 & .031 & .01 to .16 & .32 \\
\hline \multirow[t]{5}{*}{ Poland $n=306$} & Extraversion & .03 & .10 & 3.47 & .001 & .04 to .16 & -.48 \\
\hline & Agreeableness & $<.01$ & -.00 & -0.12 & .907 & -.07 to .07 & \\
\hline & Conscientiousness & $<.01$ & .00 & 0.10 & .920 & -.06 to .06 & \\
\hline & Emotional stability & .02 & .08 & 2.95 & .003 & .03 to .14 & -.61 \\
\hline & Openness to experience & $<.01$ & .01 & 0.16 & .872 & -.07 to .08 & \\
\hline
\end{tabular}

Statistical measures for significant moderators are printed in bold type

\subsection{Balanced Time Perspective as a Moderator}

We expected that the moderating effect of BTP would change the direction and/or strength of the relationship depending on the level of BTP (Baron and Kenny 1986). Moderation analyses were performed, with personality as the predictor, DBTP as the moderator, and PO as the dependent variable. We computed bootstrap-generated confidence intervals to determine the statistical significance of the interaction. In the case of significant interactions, we applied the Johnson-Neyman technique to determine the regions of significance-the value(s) of the moderator above/below which the relationship between the predictor and the outcome begins/ceases to be significant. The analysis was performed by means of PROCESS 3 software (Hayes 2018), using Model 1. We computed the moderations for each country separately. The results are presented in the Table 7.

The results show that the strength of the relationship between personality and PO depend on the level of DBTP. DBTP was a significant moderator in the relationship between extraversion and PO both in Chile and in Poland, in the relationship between openness to experience and PO in Chile, and in the relationship between emotional stability and PO in Poland. In all cases, the relationship between personality traits and PO was strong when the level of DBTP was medium or high, but at low DBTP this link was not significant. The relationship between openness to experience and PO in Chile was positive and strong only when DBTP was high. In general, this means that highly balanced individuals are less dependent on the influence of personality on PO. We used the Johnson-Neyman method as a technique for probing statistically significant interactions (Johnson and Neyman 1936). Basically, it identifies the values of the moderator for which the effect of the predictor on the outcome becomes or ceases to be significant. In Chile the relationship between extraversion and positive orientation was not significant at low values 
of the moderator, up to $-0.38 S D$. Above this value, the relationship was significant and positive. The relationship between openness to experience and positive orientation was not significant at low values of the moderator, up to $0.32 S D$. Above this value, the relationship was significant and positive. In Poland, the relationship between extraversion and positive orientation was not significant at low values of the moderator up to $-0.48 S D$. Above this value, the relationship was significant and positive. The relationship between emotional stability and positive orientation was not significant at low values of the moderator, up to $-0.61 S D$. Above this value, the relationship was significant and positive.

\section{Discussion}

Positive psychology is aimed at finding character strengths and at determining which of them are related to happiness. Our study was intended to make a further contribution to the theory of positive psychology by focusing on a new construct, PO, comprising life satisfaction, optimism, and self-esteem. The relationships were tested in three countries representing different cultures, values, and traditions. Firstly, we found the predictive power of TPs on PO even after controlling for personality traits in Poland and Chile, which supports H1. Secondly, as hypothesized (H2), BTP acted as a moderator in the relationship between personality traits and PO.

TP explained a substantial amount of variance in PO even when personality traits were controlled for (10\% in Chile and $24 \%$ in Poland). Only in the case of Hong Kong did personality traits explain a slightly higher proportion of variance in PO than TP (6 vs. 4\%). TPs added incremental value in explaining PO, the effect being similar to that obtained in the case of predicting satisfaction with life (Zhang and Howell 2011; Stolarski and Matthews 2016). These results suggested the universal role of TP and its predictive value for optimal functioning. The evaluation of oneself and one's life involved in PO depends to some extent on biological determinants (see Barnes and Pickering 1985), but also other factors should be considered, such as the attitudes towards time making up the TP profile. The important role of TP in explaining PO may stem from the nature of PO, which reflects the pattern of experience and the way of perceiving and evaluating events (Caprara et al. 2010) and therefore has a different nature compared to the five personality factors.

We found some similarities found between the investigated countries in the relationship between PO and personality traits. Of the personality traits, extraversion and emotional stability were positive predictors of PO in Chile and Poland. These findings are in line with other studies, which also showed that extraversion was the strongest predictor of life satisfaction (Joshanloo and Afshari 2011; Schimmack et al. 2004). There might be crosscultural differences, however, as shown in the recent study by Kim et al. (2018), whose results are in line with ours. Emotional stability is a salient predictor of life satisfaction and happiness, as shown in a meta-analysis of the relationships between 137 distinct personality constructs and subjective well-being (DeNeve and Cooper 1998).

Conscientiousness was a positive predictor of PO in Hong Kong and Chile. In accordance with the Confucian tradition, it is a highly valued personality trait in Chinese culture (Hofstede 2001) and a quality conducive to effective performance in Hong Kong (Moy 2006). In addition, conscientiousness was Conscientiousness may be highly valued in industrial countries - in Hong Kong and in Chile, where the economy is quite strong. For instance, Allik and McCrae (2004) found that achievement striving, dutifulness, order, and conscientiousness were positively correlated with GDP. 
As regards results concerning the relationship between PO and TP, Past Negative TP was a powerful negative predictor of PO in Chile and Poland. Similarly, in a recent study (SobolKwapinska and Jankowski 2016) conducted on a Polish sample, Past Negative TP was negatively related to PO. Porter and Peace (2007) found that negative experiences can be expected to have more lasting effects on well-being and mental health. Another possible explanation for this relationship may lie in the tendency towards ruminative thinking, which is conducive to Past Negative TP (Matthews and Stolarski 2015). We found an independent contribution of Past Positive and Present Hedonistic TPs to PO in a similar vein as Zhang and Howell (2011). Past Positive TP was a positive predictor of PO in Poland and Chile, which is in line with the results of previous research (e.g., Shmotkin and Shrira 2012). Present Hedonistic TP was a significant predictor of PO in Hong Kong and Poland. Hedonistic orientation manifests itself in seeking pleasurable experience (Feldman 2004), pleasing oneself, and providing oneself with positive stimulation, which may translate into a sense of happiness. As expected, based on previously reported findings (e.g., Przepiorka 2016; Sailer et al. 2014; Sheldon and Lyubomirsky 2006; Zimbardo and Boyd 1999, 2008), Future TP was a positive predictor of $\mathrm{PO}$ in Chile.

Our study revealed the role of BTP in predicting PO, thus supporting H2. BTP served as a moderator between personality traits and PO. Of the three countries, Hong Kong is the only one in which there were no significant moderation findings. One of the possible reasons for this may be the fact that in Chinese culture emphasis is placed on the future time perspective rather than on BTP. Education in Hong Kong is focused on preparation for the future and on individual values rather than on the present and on common goals. According to Hofstede (2001), Hong Kong has a top position in the level of long-term orientation, promoting values such as perseverance, order, thriftiness, or status observance in interpersonal relations.

It had a preventive function with regard to the effect of personality traits on PO: in other words, it played a buffering role as a moderator by decreasing the effect of personality traits on PO. This would highlight the role of BTP as a determinant of temporal self-regulation (see Zhang et al. 2013). In individuals with high BTP personality becomes less important in the evaluation of one's life, oneself, and the future, which is what constitutes PO. When we used moderation analyses, the interactions of some personality traits and DBTP predicted PO. Extraversion, openness to experience, and emotional stability differently affect PO depending on the degree of DBTP, in that sense that these personality traits may increase PO under the condition that the individual is high in unbalanced TP. The present study showed that at medium and high DBTP, the impact of some personality traits on PO became more prominent. Both in Poland and in Chile, at medium or high DBTP, we found a positive influence of extraversion on PO. A similar effect was found in the present study for openness to experience in Chile and for emotional stability in Poland. The influence of personality on PO was the strongest in individuals with high DBTP. These effect was reduced among highly balanced individuals. High level of balanced TP can counteract the effect of the personality traits on PO. This is consistent with the results obtained by Stolarski (2016), who reported that personality traits such as extraversion/neuroticism were particularly strong predictors of well-being when TP was not balanced. 


\section{Limitations and Future Directions}

The study is not free from limitations. Firstly, it was based on correlation analyses, which makes it impossible to determine the direction of effect. Secondly, the scales measuring personality traits had low reliability, its values being similar to those obtained in the original study (see Gosling et al. 2003). Although short scales are less reliable than longer ones, they have a number of advantages for research purposes. Thirdly, there was a different number of participants in each country, and there were more females in each of the three samples. Fourthly, we decided to use a long version of the ZTPI with 56 items, as this measure had already been tested and found to have good psychometric properties in these countries; it had also been used in cross-cultural comparisons (e.g., Boniwell et al. 2010; Stolarski et al. 2016). Future research should cover more countries, representing different continents for comparison. It is also advisable to investigate specific kinds of independent and interdependent self-construal and self-appraisal in each culture (Markus and Kitayama 1991).

\section{Conclusion}

The present study has shed some light on the relationship of PO with TP and personality traits. We examined more profoundly the cross-cultural differences in personality and their relationships with TP in three countries: Chile, Hong Kong, and Poland. In the cases of Chile and Poland we found the TP had incremental validity over personality traits in predicting PO. Additionally, the study revealed a moderating role of BTP in the relationship between personality and PO. It can be concluded that BTP cannot be simply reduced to a personality profile but makes its own contribution to human functioning and is an important factor in achieving the optimal level of happiness.

Important practical implications can also be expected. The significance of cross-cultural studies such as the present one stems from the fact that, to a great degree, culture influences the way people experience time (Levine 1997). Knowing the predictors of PO, we may improve the attitude towards time and thus make life more satisfying. As TP is prone to change, it is possible to work on changing the temporal profile through one's own activity as well as through therapeutic activity; therefore, the knowledge yielded by the findings of the present study will be applicable in psychological practice as well as in coaching aimed at teaching people how to manage their time in more satisfying ways.

Open Access This article is distributed under the terms of the Creative Commons Attribution 4.0 International License (http://creativecommons.org/licenses/by/4.0/), which permits unrestricted use, distribution, and reproduction in any medium, provided you give appropriate credit to the original author(s) and the source, provide a link to the Creative Commons license, and indicate if changes were made.

\section{References}

Alessandri, G., Caprara, G. V., \& Tisak, J. (2012a). A unified latent curve, latent state-trait analysis of the developmental trajectories and correlates of positive orientation. Multivariate Behavioral Research, 47(3), 341-368. 
Alessandri, G., Caprara, G., \& Tisak, J. (2012b). The unique contribution of positive orientation to optimal functioning: Further explorations. European Psychologist, 17(1), 44-54.

Allik, J., \& McCrae, R. R. (2004). Toward a geography of personality traits: Patterns of profiles across 36 cultures. Journal of Cross-Cultural Psychology, 35(1), 13-28. https://doi.org/10.1177/0022022103 260382.

Barnes, R., \& Pickering, J. (Eds.). (1985). Nature versus nurture. Lanham, MD: University Press of America.

Baron, R. M., \& Kenny, D. A. (1986). The moderator-mediator variable distinction in social psychological research: Conceptual, strategic, and statistical considerations. Journal of Personality and Social Psychology, 51(6), 1173.

Bhullar, N., Surman, G., \& Schutte, N. S. (2015). Dispositional gratitude mediates the relationship between a past-positive temporal frame and well-being. Personality and Individual Differences, 76, 52-55. https://doi.org/10.1016/j.paid.2014.11.025.

Boniwell, I., Osin, E., Linley, P. A., \& Ivanchenko, G. V. (2010). A question of balance: Time perspective and well-being in British and Russian samples. The Journal of Positive Psychology, 5(1), 24-40. https://doi.org/10.1080/17439760903271181.

Boniwell, I., \& Zimbardo, P. G. (2004). Balancing time perspective in pursuit of optimal functioning. In P. Linley \& S. Joseph (Eds.), Positive psychology in practice (pp. 165-178). Hoboken, NJ: Wiley.

Boyce, C., Wood, A., \& Powdthavee, N. (2013). Is personality fixed? Personality changes as much as "variable" economic factors and more strongly predicts changes to life satisfaction. Social Indicators Research, 111(1), 287-305. https://doi.org/10.1007/s11205-012-0006-z.

Caprara, G. V., Alessandri, G., \& Barbaranelli, C. (2010a). Optimal functioning: The contribution of self-efficacy beliefs to positive orientation. Psychotherapy and Psychosomatics, 79, 328-330.

Caprara, G. V., Alessandri, G., Eisenberg, N., Kupfer, A., Steca, P., Caprara, M. G., et al. (2012). The positivity scale. Psychological Assessment, 24(3), 701-712.

Caprara, G. V., Steca, P., Alessandri, G., Abela, J. R., \& McWhinni, C. D. (2010b). Positive orientation: Exploration on what is common to life satisfaction, self-esteem, and optimism. Epidemiology and Psychiatric Sciences, 19, 63-71.

Central Intelligence Agency (CIA). (2015). GDP-Real growth rate (\%). Retrieved March 22, 2015, from www.cia.gov.

Cohen, J. (1988). Statistical power analysis for the behavioral sciences. San Diego, CA: Academic Press.

DeNeve, K., \& Cooper, H. (1998). The happy personality: A meta-analysis of 137 personality traits and subjective well-being. Psychological Bulletin, 124(2), 197-229.

Desmyter, F., \& De Raedt, R. (2012). The relationship between time perspective and subjective wellbeing of older adults. Psychologica Belgica, 52(1), 19-38. https://doi.org/10.5334/pb-52-1-19.

Diaz-Morales, J. F. (2006). Estructura factorial y fiabilidad del Inventario de Perspectiva Temporal de Zimbardo [Factorial structure and reliability of the Zimbardo Temporary Perspective Inventory]. Psicothema, 18(3), 565-571.

Feldman, F. (2004). Pleasure and the good life: Concerning the nature, varieties and plausibility of hedonism. Oxford: Clarendon Press.

Forbes, G., Zhang, X., Doroszewicz, K., \& Haas, K. (2009). Relationships between individualism-collectivism, gender, and direct or indirect aggression: A study in China, Poland and the US. Aggressive Behavior, 35, 24-30.

Funder, D. C. (1997). The personality puzzle. New York, NY: Norton.

Garcia, D. (2014). La vie en rose: High levels of well-being and events inside and outside autobiographical memory. Journal of Happiness Studies, 15(3), 657-672. https://doi.org/10.1007/s10902-013-9443-x.

Gosling, S. D., Rentfrow, P. J., \& Swann, W. J. (2003). A very brief measure of the big-five personality domains. Journal of Research in Personality, 37, 504-528. https://doi.org/10.1037/t07016-000.

Grassi, M. C., Alessandri, G., Pasquariello, S., Milioni, M., Enea, D., Ceccanti, M., et al. (2014). Association between positivity and smoking cessation. BioMed Research International, 780146, 1-9. https://doi.org/10.1155/2014/780146.

Gross, J. J. (2008). Emotion regulation. In M. Haviland-Jones, J. M. Haviland, \& L. Feldman Barrett (Eds.), Handbook of emotions (pp. 497-512). New York: Guilford Press.

Hayes, A. F. (2018). Partial, conditional, and moderated moderated mediation: Quantification, inference, and interpretation. Communication Monographs, 85, 4-40.

Heikamp, T., Alessandri, G., Laguna, M., Petrovic, V., Caprara, M. G., \& Trommsdorff, G. (2014). Cross-cultural validation of the positivity-scale in five European countries. Personality and Individual Differences, 71, 140-145. https://doi.org/10.1016/j.paid.2014.07.012. 
Hofstede, G. (2001). Culture's consequences: Comparing values, behaviors, institutions, and organizations across nations (2nd ed.). Thousand Oaks, CA: Sage.

Huang, M., Chen, Y., \& Elfenbein, H. A. (2014). A Chinese version of the TIPI. (Unpublished manuscript).

Hwang, A., Francesco, A. M., \& Kessler, E. (2003). The relationship between individualism and collectivism, face, and feedback and learning process in Hong Kong, Singapore, and the United States. Journal of Cross-Cultural Psychology, 34(1), 72-91.

Ivanchenko, G., Osin, E., \& Leontiev, D. (2009). Perception of possibilities, subjective well-being and future time perspective. In Poster presented at the first world congress on positive psychology, June 18-21, 2009, Philadelphia, USA.

Johnson, P. O., \& Neyman, J. (1936). Tests of certain linear hypotheses and their applications to some educational problems. Statistical Research Memoirs, 1, 57-93.

Jones, B. A., Landes, R. D., Yi, R., \& Bickel, W. K. (2009). Temporal horizon: Modulation by smoking status and gender. Drug and Alcohol Dependence, 104, S87-S93.

Joshanloo, M., \& Afshari, S. (2011). Big Five personality traits and self-esteem as predictors of life satisfaction in Iranian Muslim university students. Journal of Happiness Studies, 12(1), 105-113. https://doi. org/10.1007/s10902-009-9177-y.

Kairys, A., \& Liniauskaite, A. (2015). Time perspective and personality. In M. Stolarski, N. Fieulaine, W. van Beek, M. Stolarski, N. Fieulaine, \& W. van Beek (Eds.), Time perspective theory; Review, research and application: Essays in honor of Philip G. Zimbardo (pp. 99-113). Cham: Springer. https ://doi.org/10.1007/978-3-319-07368-2_6.

Kim, H., Schimmack, U., Oishi, S., \& Tsutsui, Y. (2018). Extraversion and life satisfaction: A cross-cultural examination of student and nationally representative samples. Journal of Personality, 86(4), 604-618.

Kolstad, A., \& Horpestad, S. (2009). Self-construal in Chile and Norway Implications for cultural differences in individualism and collectivism. Journal of Cross-Cultural Psychology, 40(2), 275-281.

Laghi, F., Liga, F., Baumgartner, E., \& Baiocco, R. (2012). Time perspective and psychosocial positive functioning among Italian adolescents who binge eat and drink. Journal of Adolescence, 35(5), 1277-1284.

Laguna, M., Bąk, W., Purc, E., Mielniczuk, E., \& Oleś, P. (2014). Short measure of personality TIPI-P in a Polish sample. Roczniki Psychologiczne, 17(2), 421-437.

Laguna, M., Oleś, P. K., \& Filipiuk, D. (2011). Orientacja pozytywna i jej pomiar: Polska adaptacja Skali Orientacji Pozytywnej [Positive orientation and its measurement: A Polish adaptation of the positive orientation scale]. Studia Psychologiczne, 49(4), 47-54. https://doi.org/10.2478/v10167-010-0035-7.

Levine, R. (1997). A geography of time: The temporal misadventures of a social psychologist, or how every culture keeps time just a little bit differently. New York: Basic Books.

Lorenzo-Seva, U., \& Berge, J. M. (2006). Tucker's congruence coefficient as a meaningful index of factor similarity. Methodology, 2, 57-64. Retrieved from: https://openpsych.net/forum/attachment .php?aid=364.

Luthans, F., Youssef, C. M., \& Avolio, B. J. (2007). Psychological capital. Oxford: Oxford University Press.

Mahon, N. E., Yarcheski, T. J., \& Yarcheski, A. (1997). Future time perspective and positive health practices in young adults: An extension. Perceptual and Motor Skills, 84(3, Pt 2), 1299-1304.

Markus, H., \& Kitayama, S. (1991). Culture and the self: Implications for cognition, emotion, and motivation. Psychological Review, 98, 224-253.

Markus, H. R., \& Kitayama, S. (2003). Models of agency: Sociocultural diversity in the construction of action. In V. Murphy-Berman, J. J. Berman, V. Murphy-Berman, \& J. J. Berman (Eds.), Cross-cultural differences in perspectives on the self (pp. 18-74). Lincoln, NE: University of Nebraska Press.

Matthews, G., \& Stolarski, M. (2015). Emotional processes in development and dynamics of individual time perspective. In M. Stolarski, N. Fieulaine, W. van Beek, M. Stolarski, N. Fieulaine, \& W. van Beek (Eds.), Time perspective theory; Review, research and application: Essays in honor of Philip G. Zimbardo (pp. 269-286). Cham: Springer. https://doi.org/10.1007/978-3-319-07368-2_18.

McCrae, R. R., \& Terracciano, A. (2005). Universal features of personality traits from the observer's perspective: Data from 50 cultures. Journal of Personality and Social Psychology, 88(3), 547-561. https:// doi.org/10.1037/0022-3514.88.3.547.

Michel, W., Shoda, Y., \& Smith, R. E. (2004). Introduction to personality: Toward an integration. New York, NY: Wiley.

Miciuk, Ł. R., Jankowski, T., Laskowska, A., \& Oleś, P. (2016). Positive orientation and the five-factor model. Polish Psychological Bulletin, 47(1), 141-148.

Moy, J. W. (2006). Are employers assessing the right traits in hiring? Evidence from Hong Kong companies. International Journal of Human Resources Management, 17(4), 734-754.

Oatley, K., Keltner, D., \& Jenkins, J. M. (1996). Understanding emotions (2nd ed.). Hoboken: Blackwell Publishing. 
Osin, E., Boniwell, I., Linley, P. A., \& Ivanchenko, G. (2009). Balanced time perspective in Britain and in Russia. In Paper presented at the first world congress on positive psychology, June 18-21, 2009, Philadelphia, USA.

Oyserman, D., Coon, H. M., \& Kemmelmeier, M. (2002). Rethinking individualism and collectivism: Evaluation of theoretical assumptions and meta-analyses. Psychological Bulletin, 128(1), 3-72. https://doi. org/10.1037/0033-2909.128.1.3.

Porter, S., \& Peace, K. A. (2007). The scars of memory: A prospective, longitudinal investigation of the consistency of traumatic and positive emotional memories in adulthood. Psychological Science, 18(5), 435-441. https://doi.org/10.1111/j.1467-9280.2007.01918.x.

Przepiorka, A. (2016). What makes successful entrepreneurs different in temporal and goal-commitment dimensions? Time \& Society. https://doi.org/10.1177/0961463x15577264.

Renau, V., Oberst, U., Gosling, S. D., Rusiñol, J., \& Chamarro, A. (2013). Translation and validation of the Ten-Item-Personality Inventory into Spanish and Catalan. Aloma. Revista de Psicologia, Ciències de l'Educació i de l'Esport, 31, 85-97.

Robins, R., Tracy, J., Trzesniewski, K., Potter, J., \& Gosling, S. (2001). Personality correlates of selfesteem. Journal of Research in Personality, 35(4), 463-482.

Sailer, U., Rosenberg, P., Nima, A. A., Gamble, A., Gärling, T., Archer, T., et al. (2014). A happier and less sinister past, a more hedonistic and less fatalistic present, and a more structured future: Time perspective and well-being. Peerj. https://doi.org/10.7717/peerj.303.

Schimmack, U., Oishi, S., Furr, R. M., \& Funder, D. C. (2004). Personality and life satisfaction: A facet-level analysis. Personality and Social Psychology Bulletin, 30(8), 1062-1075. https://doi. org/10.1177/0146167204264292.

Schmitt, D. P., Allik, J., Mccrae, R. R., \& Benet-Martínez, V. (2007). The geographic distribution of Big Five personality traits: Patterns and profiles of human self-description across 56 nations. Journal of Cross-Cultural Psychology, 38(2), 173-212. https://doi.org/10.1177/0022022106297299.

Schwartz, S. H. (2004). Mapping and interpreting cultural differences around the world. In H. Vinken, J. Soeters, \& P. Ester (Eds.), Comparing cultures: Dimensions of culture in a comparative perspective (pp. 43-73). Leiden: Brill.

Sharpe, P., Martin, N., \& Roth, K. (2011). Optimism and the Big Five factors of personality: Beyond neuroticism and extraversion. Personality and Individual Differences, 51(8), 946-951.

Sheldon, K. M., \& Lyubomirsky, S. (2006). Achieving sustainable gains in happiness: Change your actions, not your circumstances. Journal of Happiness Studies, 7, 55-86.

Shmotkin, D., \& Shrira, A. (2012). Happiness and suffering in the life story: An inquiry into conflicting expectations concerning the association of perceived past with present subjective well-being in old age. Journal of Happiness Studies, 13(3), 389-409. https://doi.org/10.1007/s10902-011-9270-x.

Sircova, A., van de Vijver, F. J. R., Osin, E., Milfont, T. L., Fieulaine, N., Kislali-Erginbilgic, A., et al. (2014). A global look at time: A 24-country study of the equivalence of the Zimbardo time perspective inventory. Sage Open, 4, 1-12. https://doi.org/10.1177/2158244013515686.

Sobol-Kwapinska, M. (2016). Calm down-It's only neuroticism. Time perspectives as moderators and mediators of the relationship between neuroticism and well-being. Personality and Individual Differences, 94, 64-71. https://doi.org/10.1016/j.paid.2016.01.004.

Sobol-Kwapinska, M., \& Jankowski, T. (2016). Positive time: Balanced time perspective and positive orientation. Journal of Happiness Studies, 17(14), 1511-1528. https://doi.org/10.1007/s1090 2-015-9656-2.

Sobol-Kwapinska, M., Przepiorka, A., \& Zimbardo, P. G. (2016). Structure of time perspective in Poland: Validation and extension of the Zimbardo time perspective inventory across the ages. Time \& Society. https://doi.org/10.1177/0961463x16656851.

Steel, P., Schmidt, J., \& Shultz, J. (2008). Refining the relationship between personality and subjective well-being. Psychological Bulletin, 134, 138-161. https://doi.org/10.1037/0033-2909.134.1.138.

Stolarski, M. (2016). Not restricted by their personality: Balanced time perspective moderates wellestablished relationships between personality traits and well-being. Personality and Individual Differences, 100, 140-144.

Stolarski, M., Bitner, J., \& Zimbardo, P. G. (2011). Time perspective, emotional intelligence and discounting of delayed awards. Time \& Society, 20, 346-363.

Stolarski, M., Fieulaine, N., \& van Beek, W. (2015). Time perspective theory; Review, research and application: Essays in honor of Philip G. Zimbardo. Cham: Springer. https://doi.org/10.1007/9783-319-07368-2.

Stolarski, M., \& Matthews, G. (2016). Time perspectives predict mood states and satisfaction with life over and above personality. Current Psychology (New Brunswick, N.J.), 35(4), 516-526. 
Stolarski, M., Vowinckel, J., Jankowski, K. S., \& Zajenkowski, M. (2016). Mind the balance, be contented: Balanced time perspective mediates the relationship between mindfulness and life satisfaction. Personality and Individual Differences, 93, 27-31. https://doi.org/10.1016/j.paid.2015.09.039.

Sword, R. M., Sword, R. M., Brunskill, S. R., \& Zimbardo, P. G. (2014). Time perspective therapy: A new time-based metaphor therapy for PTSD. Journal of Loss \& Trauma, 19(3), 197-201. https://doi. org/10.1080/15325024.2013.763632.

Wang, Y., Chen, X. J., Cui, J. F., \& Liu, L. L. (2015). Testing the Zimbardo time perspective inventory in the Chinese context. PsyCh Journal, 4(3), 166-175.

World Bank Group. (2017). WDI Online [Data File]. Retrieved from the World Bank Web site at: http://data. worldbank.org/indicator/NY.GDP.PCAP.CD.

van Beek, W., Berghuis, H., Kerkhof, A., \& Beekman, A. (2011). Time perspective, personality and psychopathology: Zimbardo's time perspective inventory in psychiatry. Time \& Society, 20(3), 364-374. https ://doi.org/10.1177/0961463X10373960.

Veenhoven, R. (2015). Happiness in Chile, Hongkong, Poland (PL). World Database of Happiness, Erasmus University Rotterdam, The Netherlands. Viewed March 18 2015. http://worlddatabaseofhappiness.eur. $\mathrm{nl}$.

Zajenkowski, M., Witowska, J., Maciantowicz, O., \& Malesza, M. (2016). Vulnerable past, grandiose present: The relationship between vulnerable and grandiose narcissism, time perspective and personality. Personality and Individual Differences, 98, 102-106.

Zhang, J. W., \& Howell, R. T. (2011). Do time perspectives predict unique variance in life satisfaction beyond personality traits? Personality and Individual Differences, 50(8), 1261-1266. https://doi. org/10.1016/j.paid.2011.02.021.

Zhang, J., Howell, R., \& Stolarski, M. (2013). Comparing three methods to measure a balanced time perspective: The relationship between a balanced time perspective and subjective well-being. Journal of Happiness Studies, 14(1), 169-184.

Zimbardo, P. G., \& Boyd, J. N. (1999). Putting time in perspective: A valid, reliable individual-difference metric. Journal of Personality and Social Psychology, 77, 1271-1288.

Zimbardo, P. G., \& Boyd, J. N. (2008). The time paradox: The new psychology of time that will change your life. NewYork: Free Press.

Zimbardo, P. G., Keough, K. A., \& Boyd, J. N. (1997). Present time perspective as a predictor of risky driving. Personality and Individual Differences, 23(6), 1007-1023.

Publisher's Note Springer Nature remains neutral with regard to jurisdictional claims in published maps and institutional affiliations. 